\title{
Ensuring sustainability of software tools and services by cooperation with a research infrastructure
}

\begin{abstract}
Sustainability of project output and especially of the maintenance and further development of software is of growing concern for the research community. In the personalized medicine project $p$-medicine solutions that address this sustainability problem were developed and discussed in a workshop. They involve a number of interrelated and mutually supportive measures including the creation of a service center, building modular software, using common data standards, mutual service exchange with a research infrastructure, Open Source and fee-based software provision, joint promotion and deployment of tools in a regulated, clinical trial situation. These ideas join a nascent literature seeking to understand how project output can be put into a sustainable environment and to suggest solutions that may be useful for academic projects in general.
\end{abstract}

First draft submitted: 19 September 2015; Accepted for publication: 22 October 2015; Published online: 8 January 2015

Keywords: certification • clinical trials $\bullet$ ECRIN $\bullet$ good clinical practice $\bullet$ personalized medicine $\bullet$ research infrastructure $\bullet$ services $\bullet$ software $\bullet$ sustainability

\section{The sustainability challenge}

The problem of ensuring the sustainability of software developed in academic projects has been recognized as a major element of project outcome. It is expected that research projects should develop their own sustainability strategy for the time after the project ends. Often this is addressed by a high quality of the product, a suitable license and by building a community of potential users. To support the research software community in the UK, the Software Sustainability Institute [1] was founded to support better and more sustainable research software. An example cited by the Software Sustainability Institute is BioJS [2], an open source software project that developed visualization tools for biological data. Apart from fostering its technical experience, BioJS has established a user and developer community by means to reward contributors and to keep community members motivated. Sustainability issues of research infrastructure tools and services have been analyzed with the result that researchers have to work with software that often is not fit for purpose. Most academic software seems to have a short lifespan before it disappears, or is not updated anymore [3].

The EU commission expects that EU projects create an exit/sustainability strategy [4] outlining what will happen to project output after the end of the project. To address this challenge, EU projects tend to develop a sustainability plan and create ideas how to use products at local, national and European levels. For example, the EU projects SITEX [5], BIOCORE [6], METIS [7], GENOVATE [8] and EMBRC [9] developed strategies by creating plans for training, workshops, technical maintenance, for the inclusion of potential users, online portals and by addressing innovation issues (knowledge exchange, intellectual property rights, promotion and marketing).
Wolfgang Kuchinke, Christian Ohmann², Holger Stenzhorn ${ }^{3}$, Alberto Anguista ${ }^{4}$, Stelios Sfakianakis ${ }^{5}$, Norbert Graf ${ }^{3}$ \& Jacques Demotes ${ }^{6}$ ${ }^{1}$ Heinrich-Heine University, University Clinics, Moorenstr. 5, 40225 Düsseldorf, Germany

${ }^{2}$ ECRIN KKS Düsseldorf,

Universitätsklinikum, 40225 Düsseldorf, Germany

${ }^{3}$ Universitaet des Saarlandes, Universitätsklinikum, 66421 Homburg, Germany

${ }^{4}$ Universidad Politécnica de Madrid, Madrid, Spain

${ }^{5}$ Foundation for Research \& Technology Hellas, 71110 Heraklion, Crete, Greece ${ }^{6}$ ECRIN Office, 5 rue Watt, 75013 Paris, France

*Author for correspondence:

Tel.: +492118116142

Fax: +492118119702

kuchinke@med.uni-duesseldorf.de 
Nevertheless, most projects still lack a viable sustainability strategy that is based on a business plan and that considers the economic conditions for software maintenance and service provision $[10,11]$. In the end, projects have to take into account the strengths and weaknesses of their output. They have to match their key strengths with opportunities to create capabilities that can develop into competitive advantages in the field of their endeavors with the aim to turn advantages into opportunities for financial profits. For software projects in the area of personalized medicine the sustainability challenge is especially pronounced. Due to the necessity to employ many different software tools and embed them in an infrastructure for syntactic and semantic interoperability as well as legal compliance, software maintenance becomes complicated and expensive. This especially concerns the question, if it is sustainable to provide software for personalized medicine based on an Open Source model.

The development of Open Source software is popular among researchers and nearly all tools developed in the bioinformatics area are Open Source solutions. As example of Open Source Software that has reached a considerable distribution in the research community, Informatics for Integrating Biology and the Bedside (i2b2) [12] a data warehouse used by clinical researchers for discovery research must be mentioned. Its evolution over the past 10 years has been from a local research data solution to one disseminated more widely than any other software of the National Centers for Biomedical Computing (NCBC). A central point is that this Open Source Software is supported by a large user community that actively supports developers to enhance its capacities and functionalities. i2b2 decided against the option to reach long-term sustainability by joining a commercial partner, but plans to create a foundation as a central organization for the project. Anyway, this foundation is operated out of Harvard Medical School (MA, USA) and funded through donations [12]. The concept of a foundation has the advantage that the development of $\mathrm{i} 2 \mathrm{~b} 2$ is being influenced more strongly by academic users and that its design can be focused on academic goals instead of commercial ones. Nevertheless, although supported by a large user community, external grant funding beyond the one contributed by $\mathrm{NCBC}$ has been necessary for its maintenance. The case of $\mathrm{i} 2 \mathrm{~b} 2$ shows that sustainability problems are similar for all academic developers and that a combination of different forms of financial support including public funding must be found. We considered these ideas for our concept of mutual service provision of personalized medicine.

An additional obstacle to ensure sustainability of personalized medicine software is the intended use of the personalized medicine tools for clinical trials. Clinical trials are the scientific foundation for the advancement of medical research, but they are also complex, time consuming and expensive endeavors requiring a high degree of collaboration and the adherence to standards and best practices [13].

The EU FP7 project personalized medicine (p-medicine) [14] has created an infrastructure that facilitates the translation from current practice to personalized medicine in the area of cancer research. It has developed a multitude of tools that interact with each other and that are embedded in IT infrastructures (Table 1). For p-medicine, business opportunities emerge in the area of the development of novel, highly interconnected tools for the field of personalized medicine [15] and in their proven user friendliness [16]. To address the sustainability challenge, to identify opportunities for personalized medicine tools to create capabilities that can develop into competitive advantages and to find solutions to fill the financial gap created by the need to maintain software solutions, a joint p-medicine/ECRIN workshop was held. In June 2015, representatives from the personalized medicine project p-medicine [14] and ECRIN infrastructure $[17,18]$ gathered at the ECRIN center in Paris to examine opportunities and challenges facing the sustainability of software tools and services developed to support clinical trials in personalized medicine. The goal of the participants was to develop solutions and a concept that could ensure that the tools developed in personalized medicine become known and are employed and supported by a growing community of clinical trials professionals and researchers. Many ideas presented in this publication are based on discussions during meetings of the personalized medicine project group and the final p-medicine/ECRIN collaboration workshop in Paris.

\section{Software solutions for clinical trials in personalized medicine}

The IT infrastructure that has to be implemented and maintained to support personalized medicine is complex and comprehensive requiring many different tools (Table 1). Most of these tools can support the conduct of clinical trials in personalized medicine. Their functionality must go beyond the ones available for clinical research. Clinical trials in the area of personalized medicine require the collection of different types of data, not only clinical data, but also molecular data, images, model data from system biology and biosample data. In addition, personalized medicine is not possible without proper data protection and patient empowerment. Three necessary structures, a legal framework for data curation, integrated patient empowerment and the availability of an IT infrastructure for interoper- 
Table 1. Listing of the tools/services that are part of the personalized medicine information technology infrastructure and their relevance to support clinical trials conduct.

\begin{tabular}{|c|c|c|}
\hline Tool/service & Function & Clinical trial usage \\
\hline Portal & $\begin{array}{l}\text { Platform to provide access for clinicians, patients and researchers to tools and } \\
\text { services and to enable collaboration }\end{array}$ & \\
\hline ObTiMA & $\begin{array}{l}\text { Ontology-based clinical trial data management system to help clinicians in } \\
\text { designing and conducting clinical trials. In studies used to collect molecular } \\
\text { biological findings of patients with acute lymphatic leukemia (e.g., gene } \\
\text { expression data, genetic variation in drug metabolism) }\end{array}$ & +++ \\
\hline Oncosimulator & $\begin{array}{l}\text { Supports the clinician in the process of optimizing cancer treatment and } \\
\text { a platform for simulating data to explore cancer treatments. For the } \\
\text { simulation process, imaging data (e.g., MRI, CT, PET, among others, images), } \\
\text { histopathological data, histopathology slide images and molecular data (DNA } \\
\text { array data, selected molecular marker values or statuses, serum markers, } \\
\text { among others) are collected and processed }\end{array}$ & \\
\hline p-BioSPRE & $\begin{array}{l}\text { Enables and simplifies access to existing biobanks and the provision of own } \\
\text { biomaterial collections }\end{array}$ & ++ \\
\hline Doctor Eye & Clinically driven multimodal imaging platform & ++ \\
\hline Trial Outline Builder & $\begin{array}{l}\text { Supports clinicians when running clinical trials in the design, data collection } \\
\text { and data visualization of clinical trials }\end{array}$ & ++ \\
\hline Correlation Viewer & Explores associations between drugs, adverse events, diseases and pathways & \\
\hline Concept Explorer & $\begin{array}{l}\text { Depicts and uncovers correlations between drugs, side effects, molecular } \\
\text { targets and biomarkers }\end{array}$ & + \\
\hline Security framework & $\begin{array}{l}\text { Enables the secure sharing of patient data in compliance with applicable data } \\
\text { protection rules by providing means for authentication, authorization, auditing } \\
\text { and de-identification of data }\end{array}$ & \\
\hline $\begin{array}{l}\text { Pseudonymization/ } \\
\text { anonymization }\end{array}$ & $\begin{array}{l}\text { Service responsible for the deidentification of clinical data files (including } \\
\text { imaging data) }\end{array}$ & \\
\hline Data warehouse & $\begin{array}{l}\text { Central research repository of personalized medicine with services for } \\
\text { collecting, sharing and further elaborating annotated anonymized clinical data }\end{array}$ & + \\
\hline Ontology annotator & $\begin{array}{l}\text { Creates annotations for the translation of data in databases integrated in the } \\
\text { data warehouse }\end{array}$ & \\
\hline $\begin{array}{l}\text { Patient } \\
\text { empowerment }\end{array}$ & $\begin{array}{l}\text { Augments patient's knowledge about his/her disease and patient's } \\
\text { participation as an active partner in a shared decision process }\end{array}$ & + \\
\hline $\begin{array}{l}\text { Decision support } \\
\text { system }\end{array}$ & $\begin{array}{l}\text { Enables the clinician to access data and infer knowledge necessary to reach } \\
\text { the most accurate decision for patient outcome. The tool allows users to input } \\
\text { biomarker information and using such information the tool will formulate a } \\
\text { prediction about how a patient will respond to different treatment regimes. } \\
\text { In addition, the tool can combine various inputs, like pathological markers, } \\
\text { imaging data, among others }\end{array}$ & \\
\hline
\end{tabular}

ability play together and must therefore be maintained together. This includes the option to change processes and software based on future changes in the legislation for data protection and clinical trials. Thus, personalized medicine tools should not be seen as isolated applications, but as integral components of an IT infrastructure and their legal and ethical frameworks.

For this reason software demonstrations were presented as integrative scenarios during the workshop covering different aspects of clinical trials in person- alized medicine (Table 2). These integrative scenarios included: first, ontology-based clinical trial management system (ObTiMA) [19] for clinical data collection and biobank access; second, data integration by using a data warehouse with semantic annotation; and third, DoctorEye [20] for the sharing of DICOM images for second opinion. The demonstrations showed that it is not sufficient to install a tool at a clinical trials center, but that a lot of adaptation, configuration and training following the installation are necessary. It is a char- 
Table 2. The three integrative scenarios that were presented with relevance for clinical trials.

\begin{tabular}{|c|c|}
\hline Tools & Integrative scenarios \\
\hline ObTiMA & $\begin{array}{l}\text { ObTiMA is an ontology-based clinical trial management system for design of eCRFs and } \\
\text { for data collection in clinical trials. In the design phase, the system allows to visually } \\
\text { create trial master protocols, treatment plans and eCRFs. One of the eCRFs collects } \\
\text { data of biosamples connecting ObTiMA with the biobanking module. The phase of } \\
\text { data collection in clinical trials is supported by an easy-to-use data collection interface } \\
\text { providing additional functions, like data validation, pseudonymization and encryption }\end{array}$ \\
\hline $\begin{array}{l}\text { Data integration/ } \\
\text { analysis }\end{array}$ & $\begin{array}{l}\text { The data warehouse of } p \text {-medicine is a repository for securely storing and maintaining } \\
\text { data from different sources that is integrated semantically to enable reporting } \\
\text { and analysis of the stored data. The up loader module exports data from primary } \\
\text { sources, adapts the data to a supported format, applies an anonymization step and } \\
\text { finally stores annotated data. It was demonstrated how data is being annotated } \\
\text { and transferred to the triple store of the data warehouse that allows the storage of } \\
\text { semantic relationships }\end{array}$ \\
\hline $\begin{array}{l}\text { Sharing imaging } \\
\text { data }\end{array}$ & $\begin{array}{l}\text { The aim of the scenario is to receive a better diagnosis by including a reference } \\
\text { radiologist for a second opinion about a diagnosis based on an image. The system } \\
\text { requires a local radiologist and a reference radiologist. The reference radiologist } \\
\text { can download the image from a remote DICOM server and review it to obtain an } \\
\text { assessment. This process can be part of a clinical trial and is therefore connected to the } \\
\text { clinical trials data management system ObTiMA. In a corresponding eCRF information } \\
\text { about the trial, the references radiologist and other data is being collected with } \\
\text { ObTiMA }\end{array}$ \\
\hline & rt form; ObTiMA: Ontology-based clinical trial management system. \\
\hline
\end{tabular}

acteristic for software for personalized medicine that tools do not operate isolated but always as integrated entities together with other tools requiring means to ensure interoperability. Semantic interoperability plays an important role for all data sharing processes. Only with annotated data, interoperability is possible and data can be integrated and searched for in the kind of data warehouse that constitutes the core data store for personalized medicine. All modules of p-medicine are part of the personalized medicine platform and are connected to the data warehouse making it possible that results from different trials can be integrated in a single system. Such an integrative approach is a unique selling point for the p-medicine asystem; it goes beyond what is presently available for clinical trials.

\section{Collaboration with research infrastructures}

Already from the beginning of the p-medicine project, developing means for collaboration played an important part. In this context, three collaboration methods (networking, services/tools provision, partnership) [21] were defined. Use cases that employ these methods were created for different research infrastructures (e.g., Biobanking and Biomolecular Resources Research Infrastructure [BBMRI], European Infrastructure fir Translational Medicine [EATRIS], Distributed infrastructure for Life-Science Information [ELIXIR], European Clinical Research Infrastructure Network [ECRIN] ) and used in a survey to determine the significance of different per- sonalized medicine tools and services for these research infrastructures. For example, the use case for ECRIN determined the way personalized medicine tools may be employed in the ECRIN clinical research infrastructure $[22,23]$. The results of this survey showed that collaboration of $\mathrm{p}$-medicine with research infrastructures (e.g., virtual physiological human [VPH] [24], European Network for Cancer Research in Children and Adolescents [ENCCA] [25] BBMRI [26] and ELIXIR [27]) was not easy to achieve. Though, expressing a strong interest in collaboration with $\mathrm{p}$-medicine, research infrastructures hesitated to state concrete commitments for collaboration [28,29]. Collaboration possibilities were confined to certain issues, like support of standards and participation in board memberships.

BBMRI creates a pan-European biobanking infrastructure for biomedical and biological research that builds on already existing infrastructures, resources and technologies and that is embedded into European ethical and legal frameworks dealing with biosamples from patients and healthy persons [30]. To employ personalized medicine tools in BBMRI, for example, to use ObTiMA to manage biosamples for clinical trials, BBMRI must learn much more about this tool. It was suggested that ObTiMA should support the minimal datasets of BBMRI [31]. Nonetheless, BBMRI proposed to examine possible overlaps between BBMRI, p-medicine and ELIXIR that could be used for collaboration purposes. 
ELIXIR operates a sustainable infrastructure for biological information to support life science research and its translation to medicine [32]. For ELIXIR it is important that before beginning any collaboration, it has to be clarified, where to start the collaboration, how to organize it and who has to pay for it. In general, promising areas of collaboration are seen in the joint use of data standards. ELIXIR wants to know about the type of interfaces and standards in personalized medicine that could be employed to enable interaction between different tools.

The standards p-medicine employs to ensure interoperability and data sharing are ontologies and terminologies, such as the Logical Observation Identifier Names (LOINC) [33], terms from Gene Ontology and the Medical Dictionary for Regulatory Activities (MedDRA) [34], as well as medical classifications, like the International Classification of Diseases (ICD10) [35]. For clinical trials Systematized Nomenclature of Medicine Clinical Terms (SNOMED CT) [36] and Clinical Data Interchange Standards Consortium (CDISC) standards [37] are used.

EATRIS [38,39] provides a new development pathway, open to researchers and companies in need of support of biomedical innovations and translational medicine. Though interested in the activities of p-medicine, EATRIS has statutes that limit any participation of EATRIS to only those institutions that are listed in its official agreement. These institutions are able to support their own physical infrastructures and finance their developments. Nonetheless, EATRIS may collaborate on practical issues with p-medicine and plans to have representatives of p-medicine in its enlarged advisory board to ensure communication and harmonization.

BioMedBridges, an EU project that creates an infrastructure for data bridges between research infrastructures [40], could play a role as new enabling infrastructure by developing measures to allow interoperability between data and services in the biological, medical, translational and clinical domains including the area of research in personalized medicine. In fact, one of the five use cases of BioMedBridges covers a data bridge for personalized medicine. Participation of $\mathrm{p}$-medicine in some work packages of BioMedBridges, like the ones for the definition of interfaces, the methods for security and the use of standards were suggested. During the CRI Solutions Day [41] p-medicine presented its legal and ethical framework to the BioMedBridges group for personalized medicine.

It was suggested that the collaboration between EU projects should proceed stepwise from communication to more intensive cooperation, coordination and finally to coadunation [21]. The academic research community needs better ways to collaborate and reuse and exchange developed software tools. Especially in the area of data management and data warehousing, many similar solutions have been developed by EU projects $[41,42]$. It has now become more important to foster the joint use of software tools or the exchange of existing solutions between projects than to develop new tools. For this purpose, $\mathrm{p}$-medicine tools are built in a modular fashion and employ common data standards and ontologies. This should enable the free combination of personalized medicine tools with other, external solutions. For EU-funded projects improved cooperation capabilities should enable the exchange of resources and people as carriers of expertise between projects and infrastructures. Users of tools and tool developers should learn more efficiently from each other. The collaboration approach between projects should move from simple networking to service/tool provision and partnership [21]. To reach such a level of collaboration, common standards and business models, shared decision making and finally the sharing of staff and resources are necessary. Our concept of mutual exchange of services between p-medicine and ECRIN advances in this direction of building the basics for partnerships.

\section{Conditions for efficient collaboration with ECRIN}

Jacques Demotes (General Director ECRIN) provided the viewpoint of ECRIN, which is the viewpoint of the clinical trials community. ECRIN is an infrastructure that supports international clinical trials by providing a network of distributed groups [43]. ECRIN services include access to patients and to clinical trial expertise throughout Europe enabling international trials despite the fragmentation of health policies, legislative and funding systems. Structurally, ECRIN is based on the connection of national networks of clinical research centers and clinical trial units that provide services on the national level. In this way, each ECRIN member country builds upon its own national network of clinical trial centers. Since 2013, ECRIN is an ERIC [44], with seven countries as members and a core team in Paris.

In contrast to the rather homogeneous structure of p-medicine and its IT infrastructure, ECRIN is a distributed and thus heterogenic infrastructure harboring many different clinical trial centers that use their own software and employ their own processes. For ECRIN, it is therefore important to manage its network in a way that ensures that there is no competition between ECRIN centers and national networks, but mutual support and cooperation. For this reason, ECRIN cannot force any new software upon its many members.

Several aspects associated with the way clinical trials are conducted determine the kind of collaboration possible for ECRIN and the constraints limiting any 
employment of new software solutions. First, ECRIN is not the sponsor of clinical trials. This means that for each ECRIN trial a contract has to be concluded between ECRIN and the respective clinical trial sponsor [45]. Second, ECRIN owns no central data center, but receives support from several local ECRIN data centers. Third, ECRIN has developed a quality standard and associated procedures to certify data centers for their Good Clinical Practice (GCP) compliant data management $[46,47]$. This ECRIN Data Center Certification Program [48] applies to noncommercial clinical trials units in Europe. An ECRIN certificate can demonstrate that the corresponding center can provide safe, secure, legally compliant and efficient data management for international clinical research. Forth, as part of ECRIN-IA [49], software used by European Organization for Research and Treatment of Cancer (EORTC) for clinical trials data management has been upgraded to CDISC standards (ODM) and ECRIN requirements [50]. This software called VISTA Trials is the clinical data management system of EORTC [51], a fully integrated and web-based solution applicable to trials in all therapeutic areas. Any employment of a software solution from p-medicine in ECRIN must consider these basic conditions.

In general, ECRIN is interested in collaboration with other ESFRI infrastructures and with p-medicine. As a sustainable infrastructure ECRIN is interested in next-generation tools and wants to employ them to improve clinical trial conduct and to develop the clinical trial process further. A possibility to include p-medicine competence in an EU project is to provide a link to the CORBEL cluster project [52]. Because of the conditions mentioned above, ECRIN cannot simply commit to the use of certain tools, but is only able to recommend their use to ECRIN members. In fact, only the sponsor of a clinical trial has the authorization to choose the appropriate data management tools and in this context, ECRIN may promote the use of ObTiMA as data management tool of choice to trial sponsors.

The possibilities for collaboration between p-medicine and the ECRIN community was analyzed in the personalized medicine project with the help of requirements, use cases, value propositions [22,23]. p-medicine tools are able to support large, international GCP trials. The most widely employed clinical data management systems (CDMS) in ECRIN are MACRO and Capture System, followed by eResearch Network, CleanWeb, GCP Base [53]. Increasingly the Open Source solution OpenClinica is being used in ECRIN trials. This heterogeneity in software solutions raises questions about the interoperability. Can different products be used in a single trial and can clinical trials data be exchanged between different CDMS? The possibility of using different CDMS for the collection of data in a single trial by the joint exchange of trial data and metadata has been demonstrated [54] and p-medicine can rely on this result to advance the integration of ObTiMA with other data management solutions. ObTiMA already supports the CDISC ODM (Operational Data Model) [55] standard that allows the exchange of the entire study information between different software solutions. ODM is a platform-independent format for the interchange and archiving of clinical study data including clinical data along with its associated metadata, administrative data, reference data and audit information. In the case of software for data management in clinical trials, the CDISC standard offers besides ODM (Operational Data Model) also Clinical Data Acquisition Standards Harmonization (CDASH) [56] and SEND (Standard for Exchange of Non-clinical Data) [57]. Biomedical Research Integrated Domain Group (BRIGD) [58] is a domain analysis model for the area of protocol-driven biomedical/clinical research that allows the integration of data from clinical trials and hospital systems. Not all of these data standards are already supported by p-medicine.

For ECRIN the ObTiMA data management tool received the highest interest, followed by the biobanking tool. Both tools can be combined and used not only for trials in personalized medicine, but also for other ECRIN clinical trials. For ECRIN any novel tool to be introduced to ECRIN trials centers must show an added value for ECRIN; it must be able to integrate with already existing ECRIN tools and processes. In general, ECRIN has to deal with two conflicting constraints when choosing new software tools: personal data protection as well as the need for transparency and optimal usage of data. It was recognized that any integration of a patient empowerment tool into the ECRIN data management processes will raise problems concerning data protection and privacy protection. Nonetheless, the feature of an electronic signature for patients to sign their Informed Consents (IC) would be an important and useful feature allowing the storage and archiving of electronic IC forms.

\section{Open Source Software \& computer systems validation}

It has become standard practice for research software developed by academic researchers to be released under an open-source license. In this sense, p-medicine tools will be made freely available under an Open Source license. This should enable researchers to read, understand, modify the code and to use software for their own research purposes. In contrast to other 
Open Source projects that developed an operating system or an office suite, p-medicine cannot rely on a large potential user community. Therefore, based on the experiences gained by other Open Source projects, complementary forms of commercial licensing and fee-based charges for provided services should not be excluded by p-medicine [59]. To increase the uptake of the developed tools by researchers an easily implementable route towards adoption and opportunities for integration with other tools should be aimed at [60] . These recommendations have been considered by p-medicine project; but an additional way to increase sustainability is the validation and certification of software tools for legal compliance. In p-medicine, the significance of data protection and clinical trials regulations received special attention [61]. All computer systems used within a clinical trial operate in a regulated environment and must therefore be validated for compliance with regulations and GCP standards. This process called Computer System Validation (CSV) is a documented process for assuring that a computer system does what it is designed to do. It can guarantee that the software product is fit for purpose.

Open Source Software is known to be difficult to validate. Thus, for the validation of its tools p-medicine cannot rely on the support of a software company, but must create all documents and conduct the validation process themselves. A strategy for the validation and certification of ObTiMA and DoctorEye exists already in p-medicine [62] employing standards for GCP compliance of computerized systems used for clinical trials, like ICH-GCP [63], PIC/S [64] and GAMP [65]. In addition, validation is an area where ECRIN can contribute experience.

\section{Creation of an organizational structure to support sustainability}

Norbert Graf (coordinator of p-medicine project) described the creation of an organizational structure to further sustainability of $\mathrm{p}$-medicine output. Because there is no funding available in the project addressing sustainability after the project has ended, it was necessary to set up a kind of structure that is able to provide organizational support, as well as services, training and education that advertises the use of p-medicine tools to the research community outside of the project. Organizational support is needed, to help in the search for funding and sponsorship opportunities, provision of data protection and ethics committees, as well as establishing boards and management capabilities with the overall aim to maintain the p-medicine infrastructure and to support investigators in academic trials. This structure must also support the further development of the tools, collect feedback from users and use this feed- back for tool improvement. Even patient groups should be convinced to support personal medicine trials. These are all activities that will generate little or no revenue.

The proposed organizational structure is called Study Trial and Research Center (STaRC) [66] and is intended to go beyond p-medicine's goals and create a novel kind of innovative service center to exploit new results in clinical and basic research in combination with today's possibilities in IT enabling a new generation of clinical trials in personalized medicine. Thus, STaRC will be no simple software support center, but a center with a focus on enabling and supporting all aspects of research in personalized medicine. p-medicine members will support STaRC by becoming equity holders, by the provision of tools, services and licenses and by general support. Providing maintenance for personalized medicine tools will result in costs for STaRC and it is expected that at least in the beginning, these expenditures will exceed the costs that can be afforded by STaRC. It was discussed, if maintenance of personalized medicine tools could be sufficiently delegated to an Open Source community. For p-medicine, this seems to be an unrealistic scenario, because of the limited size of the user community of tools for personalized medicine. Thus, the classic Open Source concept of providing the entire software as Open Source, with STaRC playing a consulting/training role will not be viable. Additional sources of funding will be necessary and in consequence, STaRC as a non-profit organization will depend on additional public funding.

STaRC will be a non-profit organization, because the pressure to perform financially would move STaRC's goals toward profitable changes of the software and not to the ones best for researchers and for patients. Any nonprofit business model does have its limitations. For instance, access to start-up and operating capital will be more difficult and STaRC's capability to license software to industrial partners will be limited. Thus, the support by a large infrastructure like ECRIN that itself is sustainable may be an important help for STaRC, both for the employment of tools and for reaching out to potential users.

On the other hand, key strengths of STaRC are the possibility to provide a complete set of tools to enable any user to participate in personalized medicine. Users can also build on the strong patient focus and the integration of biobanking/imaging with data collection of p-medicine tools [16]. By association with a research infrastructure and by exchanging services with this infrastructure, $\mathrm{p}$-medicine may gain access to a large group of potential users and experts of clinical trials. Nonetheless, a major hurdle of personalized medicine should not be ignored and will hamper its widespread implementation. In personalized medicine clinical trial 
Box 1. Possibilities for the mutual exchange of services between p-medicine and European Clinical Research Infrastructure Network.

Services by STaRC

- Core components provided as free Open Source Software

- ObTiMA offered as a service (ASP, SaaS)

- ObTiMA together with SAE reporting and biobank access as a service (ASP, SaaS)

- Data warehouse as a service

- Other p-medicine tools as a service

- Integration of $\mathrm{p}$-medicine tools with other existing solutions

- Training about using personalized medicine tools (train-the-trainer principle)

- Data and privacy protection framework as service

- Development of tools for clinical trials funded by European research projects

Services by ECRIN

- Promotion of $p$-medicine tools/services, during sponsor negotiations

- Training of investigators about using personalized medicine tools, local training at sites

- Requirements generation and GCP compliance standard adaption

- Testing usability/performance in an international clinical trials setting

- Validation of $p$-medicine tools or services

- Provision of first-line support for $p$-medicine tools in clinical trials

- Extension of GCP compliance to services provision

- Certification of STaRC as ECRIN data center

- Representation of STaRC in joint projects

ECRIN: European Clinical Research Infrastructure Network; GCP: Good Clinical Practice; ObTiMA: Ontology-based clinical trial management system; STaRC: Study Trial and Research Center.

units at different hospitals are operating with different systems and it may become complicated for STaRC to adjust data exchange processes with the different requirements existing at all the hospitals involved in large trials. Though, it is unlikely that STaRC can and should be realized as a commercially profitable organization, the generated overhead by service provision may complement the public funding and may play an increasingly important part with the spreading and growing importance of personalized medicine.

\section{Sustainability through collaboration}

Of all the different ESFRI infrastructures only ECRIN conducts routinely complex clinical trials and employs for this purpose many different software solutions [53]. After experiencing the integrative scenarios of p-medicine tools during the workshop, ECRIN members expressed their interest in p-medicine to improve clinical trials. On the other hand, for ECRIN data centers it can become expensive to maintain their own data management solution including the provision of regular updates and the validation and certification of software according to the GCP standard [46]. To address this problem, p-medicine has developed a novel concept for the employment of personalized medicine tools in the research infrastructure ECRIN [23,67]. It consists of a combination of reciprocal services, complemented by the provision of Open Source and fee-based tools. This concept includes the combined provision of ObTiMA as a service $(\mathrm{SaaS})$ and as Open Source version. The
Open Source version is aimed at users that do not need the additional modules, like biobanking, imaging, adverse events reporting, data pseudonymization and data warehouse access, but are content with using only the data management functions of ObTiMA. The service version provides all additional modules and support against a charge that will be different for academic or commercial sponsors of clinical trials.

The combination of Open Source projects supported by services provided by companies that help customers with installation, configuration and troubleshooting is a well-known concept; MySQL [68] and Linux [69] are familiar examples [70]. In the area of research software for personalized medicine, the absence of a large user community, the complexity of the IT infrastructure and the multitude of necessary tools hamper the development of such service companies [71]. Especially the need to employ p-medicine tools in a regulated environment that is subject to strict rules and regulations made it necessary for us to search for a new support concept [72]. Our basic idea is to reduce the costs for using personalized medicine services by ECRIN and at the same time to disseminate its usage in the clinical trials community by employing a reciprocal exchange of services between p-medicine and ECRIN (Box 1). For this purpose, $\mathrm{p}$-medicine will provide ObTiMA and/or other tools to ECRIN and ECRIN plans to provide training, validation and support for the use of these tools and carry out the certification of STaRC as an ECRIN data center. 
In our concept, the most important aim for the personalized medicine framework must be to extend its user community and to collect experiences with the tools to create products able to withstand the conditions of international clinical trials in a regulated environment. To achieve this aim, personalized medicine tools must be employed in real clinical trial situations and ECRIN could support this aim by encouraging ECRIN trial units to test and evaluate the software. In this way, with the help of ECRIN, p-medicine tools will become known to relevant members of the European clinical research community. In addition, it was suggested to extend the collaboration to the joint search for public funding for further development and employment of personalized medicine tools. Collaboration must be organized and paid for and for this purpose, a durable equilibrium between support by public funding and fee-based charges for services must be found.

As a result of our discussions, we are convinced that only a couple of joint and interrelated activities can lead to successful support of sustainability of software developed for research purposes (Box 2). Because service provision for clinical trials is expensive, Open Source provision and fee-based income must be complemented by forms of long-term public funding and by collaboration efforts with research infrastructures. Anyway, the first step to ensure the sustainability for ObTiMA and other tools is to demonstrate that they can be used efficiently and safely in large clinical trials for collecting molecular, imaging and clinical data.

\section{Conclusion}

For EU projects ensuring the sustainability of project output is a major concern. This is especially the case when the project developed not only in a single software solution but a complete infrastructure consisting of models, polices, data protection framework and many interacting software solutions. In such cases, the step to a commercial product is very difficult. Collaboration with a research infrastructure and the mutual exchange of services is a possibility to strengthen the sustainability of project output.

We have presented the complex space of conditions and arguments that lead to decisions about ensuring sustainability for research software and hope that researchers will recognize them when thinking about their own projects. Our discussion and the suggested solutions are in line with other academic developers of software solutions for research and may be a model for similar discussions by other projects that need to find solutions for their sustainability problems.

\section{Future perspective}

The kind of complex IT infrastructure necessary to conduct research in personalized medicine requires the maintenance of the underlying legal and ethi-

\section{Box 2. Collaboration between p-medicine and European Clinical Research Infrastructure Network.}

\section{Means to enhance collaboration between personalized medicine \& ECRIN}

- p-medicine provides a reference installation for personalized medicine tools for testing by ECRIN

- Access will be provided over a special ECRIN website with additional training material

- Validation of ObTiMA for GCP compliance

- Implementation of a full CSV by ECRIN members

- Certification of STaRC as GCP compliant data center

- ECRIN is using the Open Source version of $p$-medicine tools

- $\mathrm{p}$-medicine tools are built in a modular way supporting common data standards and ontologies to enable interaction between tools

- Joint search for public funding

- p-medicine together with ECRIN are applying for appropriate calls of H2020 in 2016/2017

- The employment of personalized medicine tools will be part of these applications

- A pilot project will be set up to address a joint usage of ObTiMA and other CDMS (e.g., OpenClinica) using data exchange, or combination of the CDMS with specific modules from $p$-medicine (biobanking, images)

- The pilot project should make use of the CDISC ODM exchange standard

- Joint networking with e-infrastructures like EUDAT, RDA, EGI to further the deployment and further development of $\mathrm{p}$-medicine tools and the personalized medicine infrastructure

- Exchange of services between ECRIN and p-medicine, joint evaluation and validation of services

- Joint promotion of $\mathrm{p}$-medicine tools and infrastructure to get clinical trial sponsors interested and to further the development of clinical trials in personalized medicine in general

- Build a user community that is involved in the use of $\mathrm{p}$-medicine tools, but also in the improvement of the tools and the support of research in personalized medicine

CDISC: Clinical Data Interchange Standards Consortium; CDMS: Clinical Data Management System; CSV: Computer System Validation; ECRIN: European Clinical Research Infrastructure Network; EGI: European Grid Infrastructure; EUDAT: Research Data Services, Expertise and Technology Solutions; GCP: Good Clinical Practice; ObTiMA: Ontology-based clinical trial management system; ODM: Operational Data Model; RDA: Research Data Alliance. 
cal framework and a multitude of interacting tools and services. The maintenance of such infrastructures cannot be achieved by a single project alone, but requires collaboration and partnerships between project partners, infrastructures and expert centers. Before developing a new software solution, developers should check whether a reuse of existing tools and services is possible. Newly developed software should be built in a modular way for interaction with other tools and for integration in e-infrastructures that may contribute to the maintenance and service provision of these tools. An active approach to include research infrastructures and e-infrastructures should be considered. Especially EUDAT [73] and RDA [74] are building a generic data exchange layer and a generic data sharing framework. In the case of EUDAT, this is the Collaborative Data Infrastructure (CDI) for pan-European solutions. In this way, in future all developed tools can be part of this collaboration framework. They should support and complement each other. The involvement of large e-infrastructures in the service provision and maintenance of newly developed tools will have a positive effect on the sustainability of research software developed by academic centers and will thereby contribute to advance the field of personalized medicine. In any case, the EU should provide funding for these efforts.

\section{Financial \& competing interests disclosure}

The research leading to the results presented and the software developed have received funding from the European Community's Seventh Framework Programme (FP7/2007-2013) under grant agreement No. 270089. The authors have no other relevant affiliations or financial involvement with any organization or entity with a financial interest in or financial conflict with the subject matter or materials discussed in the manuscript apart from those disclosed.

No writing assistance was utilized in the production of this manuscript.

\section{Open access}

This article is distributed under the terms of the Creative Commons Attribution License 4.0 which permits any use, distribution, and reproduction in any medium, provided the original author(s) and the source are credited. To view a copy of the license, visit http://creativecommons.org/licenses/by/4.0/

\section{Executive summary}

\section{The sustainability challenge for IT solutions}

- Personalized medicine requires the implementation of a complex IT infrastructure able to process clinical data, molecular data, images and models, and biosamples data.

- The guarantee of sustainability of such an infrastructure is a challenge for all academic centers.

Software solutions for clinical trials in personalized medicine

- Examples for integrative software solutions for personalized medicine are: ontology-based clinical trial management system for clinical data collection and biobank access; integration of a data warehouse with semantic annotation; DoctorEye for sharing DICOM images for second opinion.

Collaboration with research infrastructures

- Of all questioned research infrastructures only European Clinical Research Infrastructure Network (ECRIN) provided the best prerequisites for collaboration and employment of personalized medicine tools. Cooperation of projects and infrastructures is still limited to the level of data standards and interface specifications.

- Personalized medicine tools of the p-medicine project will be employed in large, international Good Clinical Practice trials.

\section{Creation of an organizational structure}

- Central to the management of sustainability is the creation of an organizational structure; it should be able to provide support, service, training and education and to maintain the personalized medicine infrastructure.

\section{The ECRIN point of view}

- ECRIN is interested in next-generation tools and wants to employ them to improve clinical trial conduct and to develop the clinical trial process further.

- ECRIN cannot commit to the use of certain tools, but is only able to recommend the use of a tool to its member clinical trials centers.

\section{Sustainability through collaboration}

- A mutual benefit by the reciprocal exchange of services can be achieved between ECRIN and p-medicine in the area of clinical trials.

- ECRIN will support the validation of software for Good Clinical Practice compliance.

Sustainability of software solutions developed by academic centers

- For academic software development, it is necessary to use simultaneously several financial sources, but also to use new forms of support such as that provided by the mutual exchange of services. A durable equilibrium between support by public funding, fee-based charges for services and commercial licensing must be found. 


\section{References}

Papers of special note have been highlighted as:

- of interest; $\bullet$ of considerable interest

1 Crouch S, Chue Hong N, Hettrick S et al. The Software Sustainability Institute: changing research software attitudes and practices. Comput. Sci. Eng. 15(74), 74-80 (2013).

2 Yachdav G, Goldberg T, Wilzbach S et al. Cutting edge: anatomy of BioJS, an open source community for the life sciences. eLife 4, e07009 (2015).

3 Hanganu G. Sustainability lessons for research infrastructure. OSSWatch, Briefing notes, 9 August 2010. University of Oxford, UK (2010).

http://oss-watch.ac.uk/resources

4 Managing projects. Exit/sustainability strategy mandated by the European Commission for EU projects. EU Commission, Brussels, Belgium (2012).

http://ec.europa.eu/chafea/management

5 SITEX Deliverable 6.1: Conditions for establishing a sustainable expertise network, Cordis (2014). http://cordis.europa.eu/fp7/euratom-fission

6 BIOCORE Deliverable D7.4: Final assessment of the economic, social/legal/political sustainability of the BIOCORE system, biocore-europe (2013). www.biocore-europe.org

7 METIS Deliverable 6.5: Sustainability \& Exploitation Plan, metis-project (2015). www.metis-project.org/resources

8 GENOVATE Del 8: Dissemination and Sustainability Strategy, genovate (2013). www.genovate.eu/media/genovate

9 EMBRC Deliverable D10.5: Mutualising access with other ESFRI infrastructures, EMBRC (2013). www.embrc.eu/sites/default/files/D10.5.pdf

10 Hilty L, Lohmann W, Huang E. Sustainability and ICT - an Politeia 104, overview of the field. Politeia 104 13-28 (2011).

- This article gives an overview of different approaches of using information technology to achieve sustainability. Important is that it refers to the fact that technological efficiency alone will not produce sustainability, but a combination of efficiency and sufficiency strategies is most effective.

11 Mahaux M, Heymans P, Saval G. Discovering Sustainability Requirements. In: Requirements Engineering: Foundation for Software Quality. Lecture Notes in Computer Science (Vol. 6606). Springer, Berlin, Heidelberg, Germany (2011)

12 Murphy SN, Wilcox A. Mission and Sustainability of Informatics for Integrating Biology and the Bedside (i2b2), eGEMs 2(2), 1074-1083 (2014).

13 Kuchinke W, Ohmann C. A technical introduction to the basic requirements of clinical trials. EJHP Practice 15(5), 20-22 (2009).

14 P-medicine project. The full title of the project is "From data sharing and integration via VPH models to personalized medicine" (2015)

http://p-medicine.eu

15 Hamburg MA, Collins FS. The path to personalized medicine. N. Engl. J. Med. 363, 301-304 (2010).
- A very motivating article how medical treatments will be improved by personalized medicine. The authors compare the recent development of personal medicine infrastructure with the building of the national highway system and expect that doctors and patients will navigate these roads to achieve better medical outcomes and better health.

16 Rossi S, Christ-Neumann ML, Rüping S et al. P-medicine: from data sharing and integration via VPH models to personalized medicine. Ecancer 5, 218-224 (2011).

17 The European Clinical Research Infrastructure Network (ECRIN) (2015). www.ecrin.org/en/

18 Demotes-Mainard J, Ohmann C. European Clinical Research Infrastructures Network: promoting harmonisation and quality in European clinical research. Lancet 365(9454), 107-108 (2005).

19 Stenzhorn H, Weiler G, Brochhausen M et al. The ObTiMA system - ontology-based managing of clinical trials. Stud. Health Technol. Inform. 160(Pt 2), 1090-1094 (2010).

•- The authors present ontology-based clinical trial management system, a clinical data management system that helps researchers to collect and manage the large amount of data generated in clinical trials. The interoperability issues of such a system are addressed by using ontology and semantic data mediation. Ontologybased clinical trial management system is composed of a component for setting-up clinical trials, another for handling patient data during trials and additional modules for biosamples and adverse events data.

20 Karatzanis I, Marias K, Sakkalis V. Dr Eye. J. Clin. Bioinformat. 5(Suppl. 1), S21 (2015).

21 Karakoyun T, Kuchinke W, Ohmann C, Auer HJ. A scenario-driven approach to enable collaboration among research infrastructures and initiatives. In: $e$ Challenges e-2012 Conference Proceedings. Cunningham P, Cunningham $\mathrm{M}$ (Eds). IIMC International Information Management Corporation Ltd, Dublin, Ireland (2012).

22 Deliverable No. D6.1: report on use cases, scenarios, user needs, tools, interoperability issues for the ECRIN community. p-medicine (2014) http://p-medicine.eu/fileadmin/p-medicine

23 Deliverable No. D6.2: Evaluation report of the usability of p-medicine tools within the ECRIN infrastructure. P-medicine (2014).

24 Virtual Physiological Human (VPH) Institute. www.vph-institute.org/

25 European Network for Cancer Research in Children and Adolescents. www.encca.eu/

26 Biobanking and BioMolecular resources Research Infrastructure. http://bbmri-eric.eu/

27 ELIXIR, Wellcome Trust Genome Campus, Hinxton, UK. www.elixir-europe.org/

28 Deliverable No. D2.3: Specification of the required integration with other research infrastructures. p-medicine (2014).

http://p-medicine.eu/fileadmin/p-medicine 
29 Kuchinke W, Karakoyun T, Ohmann C. Collaboration between Research Initiatives and Infrastructures for Personalized Medical Research: an Underestimated Challenge. In: eChallenges e-2012 Conference Proceedings. Cunningham P, Cunningham M (Eds). IIMC International Information Management Corporation Ltd, Dublin, Ireland, (2012).

30 Van Ommen GJB, Törnwall O, Bréchot C et al. BBMRIERIC as a resource for pharmaceutical and life science industries: the development of biobank-based Expert Centres. Eur. J. Hum. Genetics. 23(7), 893-900 (2015).

31 Norlin L, Fransson MN, Eriksson $\mathrm{M}$ et al. A minimum data set for sharing biobank samples, information, and data: MIABIS. Biopreserv. Biobank. 10(4), 343-348 (2012).

32 Crosswell LC, Thornton JM. ELIXIR: a distributed infrastructure for European biological data. Trends Biotechnol. 30 (5), 241-242 (2012).

33 Logical Observation Identifier Names (LOINC) is a universal code system for tests, measurements, and observations. https://loinc.org/

34 Medical Dictionary for Regulatory Activities (MedDRA) is a rich and specific standardised medical terminology to facilitate sharing of regulatory information for medical products used by humans. www.meddra.org/

35 International Classification of Diseases (ICD) is a standard diagnostic tool for epidemiology, health management and clinical purposes. www.who.int/classifications/icd/en/

36 SNOMED CT is a comprehensive and precise clinical health terminology. www.ihtsdo.org/snomed-ct

37 Clinical Data Interchange Standards Consortium (CDISC). CDISC open data standards have been developed to improve the quality, efficiency and cost effectiveness of clinical research processes. www.cdisc.org/standards-and-implementations

38 European Infrastructure for Translational Medicine. www.eatris.eu/

39 Becker R, van Dongen GA. EATRIS, a vision for translational research in Europe. J. Cardiovasc. Transl. Res. 4(3), 231-237 (2011).

40 BioMedBridges is a joint effort of twelve biomedical sciences research infrastructures. www.biomedbridges.eu/

41 Kuchinke W, Karakoyun T, Gengler S, Ohmann C. First Clinical Research Informatics (CRI) Solutions Day: advanced IT support from EU projects for clinical trials. J. Clin. Bioinformatics 5(Suppl. 1), A1 (2015). www.jclinbioinformatics.com/content/5/S1/A1

-. This publication presents the results of the "First Clinical Research Informatics Solutions Day.” Many European Strategy Forum for Research infrastructures (ESFRI) provided insights into their approach for interoperability and sustainability; newly developed software tools were presented and abstracts about the presentations exist in the appendix of the publication.
42 Kuchinke W, Karakoyun T. Pathway to improved data management in clinical trials. Clin. Invest. 4(6), 501-505 (2014).

43 Demotes-Mainard J, Kubiak C. European perspective - the European clinical research infrastructures network. Ann. Oncol. 22(Suppl. 7), vii44-vii49 (2011).

44 European Research Infrastructure Consortium (ERIC) (2015). An ERIC is a legal entity. ec.europa.eu/research/infrastructures

$45 \mathrm{ICH}$, harmonization for better health. www.ich.org/home.html

•• The International Council for Harmonisation of Technical Requirements for Pharmaceuticals for Human Use provides many Guidelines for clinical trials conduct. The Guideline "ICH E6: Good Clinical Practice: Consolidated guideline, CPMP/ICH/135/95" forms the basis for Good Clinical Practice (GCP) compliance and provides all relevant definitions for this topic. Important is the sponsor definition (GCP 1.53): "Sponsor" is an individual, company, institution, or organization which takes responsibility for the initiation, management, and/or financing of a clinical trial. (GCP 1.54): "Sponsor-Investigator" is an individual who both initiates and conducts, alone or with others, a clinical trial, and under whose immediate direction the investigational product is administered to, dispensed to, or used by a subject.

46 Ohmann C, Kuchinke W, Canham S et al. ECRIN Working Group on Data Centres. Standard requirements for GCPcompliant data management in multinational clinical trials. Trials 12(1), 85-94 (2011). www.trialsjournal.com/content/12/1/85

-. This is the document that introduces the European Clinical Research Infrastructure Network (ECRIN) standard for GCP compliant data management to the research community [47]; is a recent update of the standard. The list of requirements is provided as supplementary file.

47 Ohmann C, Canham S, Cornu C et al. Revising the ECRIN standard requirements for information technology and data management in clinical trials. Trials 14, 97-107 (2013).

48 ECRIN Data Centre Certification (2015). www.ecrin.org/en/tools/data-centre-certification

49 European Clinical Research Infrastructures Network - Integrating Activity (ECRIN-IA) (2015). http://82.198.66.209

50 Gilotay C, Lejeune S. VISTA Trials. J. Clin. Bioinformat 5(Suppl. 1), 54 (2015). www.biomedcentral.com/content

- The authors present VISTA trials, a professional clinical data management system developed by the European Organisation for Research and Treatment of Cancer. The development of VISTA trials incorporates many years of experiences with oncological clinical trials by the European Organisation for Research and Treatment of Cancer. It is provided as a fully integrated and web-based solution.

51 VISTA Trials. www.vistatrials.org/ 
52 INFRADEV-4- 2014-2015 - Implementation and operation of cross-cutting services and solutions for clusters of ESFRI and other relevant research infrastructure initiatives (2015). http://cordis.europa.eu/project/rcn/197885_en.html

- Coordinated Research Infrastructures Building Enduring Life-Science Services will establish a collaborative framework of shared services between the European Strategy Forum for Research infrastructure Biological and Medical Research Infrastructures that transform the European research community from discovery of basic biological mechanisms to applied medical translation - through the provision of a unified interface, aligned services and coordinated user access to a range of advanced technology platforms. One component of the Coordinated Research Infrastructures Building Enduring Life-Science Services will be the development of a data repository for patient-based clinical trials data.

53 Kuchinke W, Ohmann C, Yang Q et al. Heterogeneity prevails: the state of clinical trial data management in Europe - results of a survey of ECRIN centres. Trials 11(1), 79-89 (2010).

www.trialsjournal.com/content/11/1/79

54 Kuchinke W, Wiegelmann S, Verplancke P, Ohmann C. Extended cooperation in clinical studies through exchange of CDISC metadata between different study software solutions. Methods Inf. Med. 45(4), 441-446 (2006).

-• Using the CDISC standard Operational Data Model, the authors demonstrated that data and metadata of a complete clinical trial could be transferred from one data management system to another, different type of data management system. This result has important consequences for using different software tools in a single trial and for enhancing the collaboration of trial data management.

55 The CDISC Operational Data Model (ODM) was created to facilitate the regulatory-compliant acquisition, archive and interchange of metadata and data for clinical research studies (2015). www.cdisc.org/odm

56 Clinical Data Acquisition Standards Harmonization (CDASH) describes the basic recommended data collection fields for 18 domains, including demographics, adverse events, and other domains that are common in clinical research (2015).

www.cdisc.org/cdash

57 Standard for Exchange of Nonclinical Data (SEND) (2015). www.cdisc.org/send

58 Biomedical Research Integrated Domain Group (BRIDG) Model provides a shared view of the dynamic and static semantics for the domain of basic, pre-clinical, clinical, and translational research and its associated regulatory artefacts (2015). www.bridgmodel.org/

59 Hong NC, Hettrick S, Katz DS. The price of open-source software - a joint response. Blog: 27 Aug 2015. Software Sustainability Institute, Edinburgh, UK (2015). www.software.ac.uk/blog/
60 Ingram D, Arikan SS. The evolving role of Open Source software in medicine and health services. Technol. Innov. Manag. Rev. 2013 32-39 (2013).

61 Deliverable No. D9.1: Report of regulatory and international aspects of the clinical trials. p-medicine (2014). http://p-medicine.eu/fileadmin/p-medicine

62 Deliverable No. 9.3: report on the validation and certification of ObTiMA and DoctorEye. p-medicine. http://p-medicine.eu/fileadmin/p-medicine

63 ICH Expert Working Group: Guideline for Good Clinical Practice E6(R1), Current Step 4 version. Presented at: International conference on harmonisation of technical requirements for registration of pharmaceuticals for human use (ICH). Geneva, Switzerland, 10 June 1996.

64 Good Practices for Computerised Systems in Regulated GXP Environments, PI 011-3 (PIC/S Guidance), Pharmaceutical Inspection Co-Operation Scheme, Geneva Switzerland (2007). www.picscheme.org/pdf/27

65 GAMP $^{\circledR}$ 5: A Risk-Based Approach to Compliant GxP Computerized Systems. International Society for Pharmaceutical Engineering, Inc. (ISPE) Tampa, Florida, USA (2008).

66 Study, Trial and Research Centre. http://eu-starc.eu/

67 Kuchinke W, Karakoyun T, Ohmann C. Integration of Data Services to Enhance Clinical Trial Data Management and GCP (Good Clinical Practice) Compliance at ECRIN Data Centres. In: eChallenges e-2013 Conference Proceedings. Cunningham P, Cunningham M (Eds). IIMC International Information Management Corporation Ltd, Dublin, Ireland, (2013).

- The authors suggest a novel way of 'deep integration' of p-medicine tools as a service with the ECRIN network based on reciprocal exchange of services provided by p-medicine and by ECRIN; requirements, training, usability tests employing infrastructure of national hubs (EU Correspondents) and support in local languages.

$68 \mathrm{MySQL}$ is a very popular database management system. http://dev.mysql.com/

69 Linux is the best-known open source operating system. www.linuxfoundation.org/

70 Kogut B, Metiu A. Open-source software development and distributed innovation. Oxf. Rev. Econ. Policy. 17(2), 248-264 (2001)

71 Feller J. Perspectives on Free and Open Source Software. The MIT Press, Cambridge, MA, USA (2005)

72 Chapman R. Regulatory issues dealing with Open Source software. Presented at: The OSS-MHS Workshop Arlington, VA, USA, 23 September, 2011.

73 EUDAT. http://eudat.eu/

74 RDA. Research Data Alliance. https://rd-alliance.org/ 